\title{
A Heat Transfer Problem of Viscous Liquid in Porous Media
}

\author{
K. Moinuddin \\ Faculty of Mathematics, Maulana Azad Nation Urdu University, Gachibowli, Hyderabad, Telangana, India \\ *Corresponding Author: K. Moinuddin, Faculty of Mathematics, Maulana Azad Nation Urdu University, \\ Gachibowli, Hyderabad, Telangana, India
}

\begin{abstract}
Steady flow of a viscous fluid of finite depth in a porous medium over a fixed horizontal, impermeable bottom is considered in this paper. Exact solutions of Momentum and Energy equations are obtained when the temperatures on the fixed bottom and on the free surface are prescribed. Velocity, Mean velocity, Temperature and MeanTemperature in the flow region have been obtained. The cases of large and small values of porosity coefficient have been obtained as limiting cases. The results are illustrated graphically.
\end{abstract}

Keywords: Viscous fluid, Velocity, Mean Velocity, Temperature, Mean Temperature

\section{INTRODUCTION}

Forced convective flows through porous and non porous channels for a variety of geometries was examined by Raghava Charyulu [1] and Satyanarayana Raju [2] . Md.Ameenuddin and N.Ch.Pattabhi Ramacharyulu [3] studied steady flow of a second order slightly viscous fluid through a porous medium between two horizontal permeable plates in relative motion in the year 2011. Jaweed Ameen[4] studied some investigations on fluid flows through generalized porous media. Raptis et.al [5] examined an oscillary flow through a porous medium in the presence of free convective flow.

In this paper the convective flow of a viscous liquid of viscosity $\mu$ and of finite depth $\mathrm{H}$ through a porous medium of porosity coefficient ' $\mathrm{k}$ ' ' over a fixed impermeable bottom is investigated. The flow is generated by a constant pressure gradient parallel to the fixed bottom plate. The momentum equation considered is the generalized Darcy's law proposed by Yama Moto and Iwamura [6] which takes into account the convective acceleration and the Newtonian viscous stresses in addition to the classical Darcy force. The basic equations of momentum and energy are solved to give exact expressions for velocity and temperature distributions. Employing, the flow rate, mean velocity, mean temperature, mean mixed temperature and the nusselt numbers at the fluid boundaries have been obtained and illustrated graphically.

The cases of 1 . high porosity and 2. low porosity are also discussed.

\section{Mathematical Formulation}

Consider the steady forced convective flow of a viscous liquid through a porous medium of viscosity coefficient $\mu$ and of finite depth (H) over a fixed horizontal impermeable bottom. The flow is generated by a constant horizontal pressure gradient parallel to the bottom. Further the bottom is kept at a constant temperature $T_{0}$ and the free surface is exposed to atmospheric temperature $T_{1}$. 


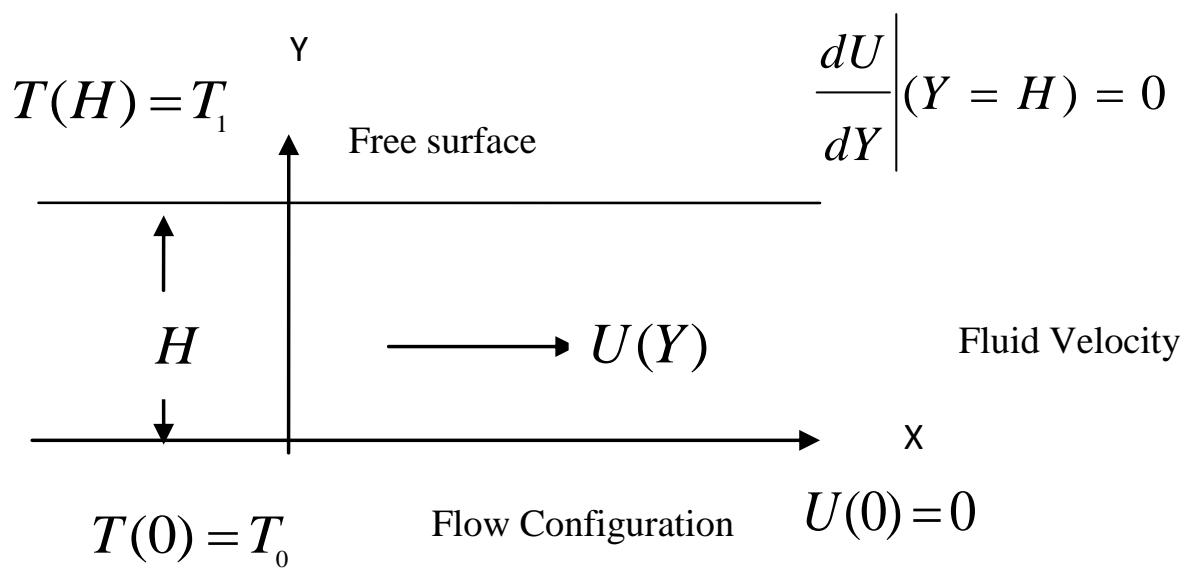

With reference io a recianguiar Cartesian co-ordinates system wıи uाe ungin ' $\mathrm{O}$ ' on the bottom, $\mathrm{X}$ axis in the flow direction (that is parallel to the applied pressure gradient).The $\mathrm{Y}$-axis vertically upwards, the bottom is represented as $\mathrm{Y}=0$ and the free surface as $\mathrm{Y}=\mathrm{H}$. Let the flow be characterized by a velocity $\mathrm{U}=(\mathrm{U}(\mathrm{Y}), 0,0)$. This choice of velocity evidently satisfies the continuity equation $\nabla . U=0$ where $\mathrm{U}$ is the fluid velocity vector. Further let $\mathrm{T}(\mathrm{Y})$ denotes the temperature distribution.

\section{BASIC EQUATIONS}

Let the convective flow be calculated by the velocity field $\mathrm{U}=(\mathrm{U}(\mathrm{Y}), 0,0)$ and the temperature $\mathrm{T}(\mathrm{Y})$. This choice of the velocity satisfies the continuity equation $\nabla . U=0$

The Momentum Equation is

$$
-\frac{\partial P}{\partial X}+\mu \frac{d^{2} U}{d Y^{2}}-\mu \frac{U}{k^{*}}=0
$$

and the Energy Equation is

$$
\rho c U \frac{\partial T}{\partial X}=K \frac{d^{2} T}{d Y^{2}}+\mu\left(\frac{d U}{d Y}\right)^{2}
$$

In the above equations $\rho$ is the fluid density, $\mathrm{k}^{*}$ the coefficient of porosity of the medium, $\mathrm{c}$ is the specific heat, $\mathrm{K}$ the thermal conductivity of the fluid and $\mathrm{P}$ the fluid pressure.

\section{Boundary Conditions:}

Since the bottom is fixed, $U(0)=0$

At the free surface shear stress vanishes

$$
\mu \frac{d U}{d Y}=0 \text { at } \mathrm{Y}=\mathrm{H}
$$

$$
\text { Also } \mathrm{T}(0)=\mathrm{T}_{0} \quad[5 \mathrm{a}] \quad \text { and } \quad \mathrm{T}(\mathrm{H})=\mathrm{T}_{1}
$$

where $T_{0}$ is the bottom temperature and $T_{1}$ is the atmosphere temperature.

In terms of the non-dimensional variables defined as:

$\mathrm{Y}=\mathrm{ay} ; \mathrm{X}=\mathrm{ax} ; \mathrm{H}=\mathrm{ah} ; U=\frac{\mu u}{\rho a^{2}} ; P=\frac{\mu^{2} p}{\rho a^{2}} ; \quad T=T_{0}+\left(T_{1}-T_{0}\right) \theta ; \operatorname{Pr}=\frac{\mu c}{k} ; k^{*}=\frac{a^{2}}{\alpha^{2}} ;$

$E=\frac{\mu^{3}}{\rho^{2} a^{2} K\left(T_{1}-T_{0}\right)} ;-\frac{\partial P}{\partial X}=\frac{\mu^{2} c_{1}}{\rho a^{3}}\left(c_{1}=-\frac{\partial p}{\partial x}\right) \quad$ and $\frac{\partial T}{\partial X}=\frac{\left(T_{1}-T_{0}\right)}{a} c_{2}$ 
where $c_{2}=\frac{\partial \theta}{\partial x}$

where ' $a$ ' is some standard length, the basic field equations (2), (3 ) can be rewritten as follows:

Momentum Equation:

$$
\frac{d^{2} u}{d y^{2}}-\alpha^{2} u=-c_{1}
$$

[7] and

Energy Equation:

$$
\frac{d^{2} \theta}{d y^{2}}=P_{r} c 2^{u-E\left(\frac{d u}{d y}\right)^{2}}
$$

together with the boundary conditions

For the velocity $\mathrm{u}(0)=0$ and $\quad \frac{d u}{d y}=0 \quad$ at $\mathrm{y}=\mathrm{h}$

and for the temperature $\theta(0)=0$ and $\theta(h)=1$

The momentum equation together with the related boundary conditions yield the velocity distribution:

$$
u(y)=\frac{c_{1}}{\alpha^{2}}\left(1-\frac{\cosh \alpha(h-y)}{\cosh \alpha h}\right)
$$

The energy equation satisfying the boundary conditions yields the temperature distribution:

$$
\begin{aligned}
& \theta(y)=\frac{y}{h}+\frac{P_{r} c_{1} c_{2}}{\alpha^{2}}\left[\frac{(h-y)}{h \alpha^{2}}-\frac{y(h-y)}{2}+\frac{1}{\alpha^{2} \cosh \alpha h}\left(\frac{y}{h}-\cosh \alpha(h-y)\right)\right]+ \\
& \frac{E c_{1}^{2}}{2 \alpha^{2} \cosh ^{2} \alpha h}\left[\frac{(h-y) \cosh (2 \alpha h)}{4 \alpha^{2} h}-\frac{y(h-y)}{2}+\frac{1}{4 \alpha^{2}}\left(\frac{y}{h}-\cosh 2 \alpha(h-y)\right)\right]
\end{aligned}
$$

The flow rate in the non-dimensional form is

$$
q=\int_{0}^{h} u(y) d y=\frac{c_{1}}{\alpha^{2}}\left(h-\frac{\tanh \alpha h}{\alpha}\right)
$$

The mean velocity in the non-dimensional form is

$$
\frac{1}{h} \int_{0}^{h} u(y) d y=\frac{c_{1}}{h \alpha^{2}}\left(h-\frac{\tanh \alpha h}{\alpha}\right)
$$

Further the mean temperature in non-dimensional form is given by

$$
\bar{\theta}=\frac{1}{{ }_{h}} \int_{0}^{h} \theta d y
$$




$$
\begin{aligned}
& =\frac{1}{2}+\frac{P_{r} c_{1} c_{2}}{\alpha^{2}}\left(\frac{-h^{2}}{12}+\frac{1}{2 \alpha^{2}}+\frac{1}{2 \alpha^{2} \cosh \alpha h}-\frac{\tanh \alpha h}{h \alpha^{3}}\right)- \\
& \frac{E c_{1}^{2}}{2 \alpha^{2} \cosh ^{2} \alpha h}\left(\frac{-1}{8 \alpha^{2}}+\frac{h^{2}}{12}-\frac{\cosh 2 \alpha h}{8 \alpha^{2}}+\frac{\sinh 2 \alpha h}{8 h \alpha^{3}}\right)
\end{aligned}
$$

\subsection{CASE 1:}

Fluid flow in a medium with high porosity i.e flow for small values of $\alpha$ or large values of the porosity coefficient $k^{*}$

Neglecting terms of $\alpha$ higher than $O\left(\alpha^{2}\right)$ we get

Velocity: $u(y)=c_{1}\left\{\frac{\left(2 h y-y^{2}\right)}{2}-\frac{\alpha^{2}}{24}\left(8 h^{3} y-4 h y^{3}+y^{4}\right)\right\}$

Mean velocity $\bar{u}=\frac{1}{h} \int_{0}^{h} u d y=\frac{c_{1} h^{2}}{15}\left(5-2 \alpha^{2} h^{2}\right)$

\section{Temperature:}

$$
\begin{aligned}
& \theta(y)=\frac{y}{h}+\frac{P_{r} c_{1} c_{2}}{720}\left[\left(120 h y^{3}-90 h^{3} y-30 y^{4}\right)-\alpha^{2}\left(h^{6}-36 h^{5} y+40 h^{3} y^{3}-6 h y^{5}+y^{6}\right)\right]+ \\
& \frac{E c_{1}^{2}}{8640}\left[720\left(3 h^{3} y-6 h^{2} y^{2}+4 h y^{3}-y^{4}\right)-\alpha^{2}\left(\begin{array}{l}
791 h^{6}-576 h^{5} y+2160 h^{4} y^{2}+ \\
960 h^{3} y^{3}+720 h^{2} y^{4}-576 h y^{5}+96 y^{6}
\end{array}\right)\right]
\end{aligned}
$$

\section{Mean temperature:}

$$
\bar{\theta}=\frac{1}{h} \int_{0}^{h} \theta d y=\frac{1}{2}-\frac{p_{r} c_{1} c_{2} h^{4}}{5040}\left(147+575 \alpha^{2} h^{2}\right)+\frac{E c_{1}{ }^{2} h^{4}}{210960}\left(5274+907 \alpha^{2} h^{2}\right)
$$

\subsection{CASE 2. For large values of $\alpha$ i.e for low porosity}

For large $\alpha \sinh \alpha h \approx \frac{e^{\alpha h}}{2} ; \cosh \alpha h \approx \frac{e^{\alpha h}}{2} ; \tanh \alpha h \approx 1$ and neglecting the terms of $O\left(\frac{1}{\alpha^{3}}\right)$ we get

Velocity: $u(y)=\frac{c_{1}}{\alpha^{2}}\left(1-e^{-\alpha y}\right)$

Mean velocity: $\bar{u}=\frac{c_{1}}{\alpha^{2}}$

Temperature: $\theta(y)=\frac{y}{h}-\frac{p_{r} c_{1} c_{2}(h-y) y}{2 \alpha^{2}}$ 
Mean Temperature: $\bar{\theta}=\frac{1}{h} \int_{0}^{h} \theta d y=\frac{1}{2}-\frac{p c c_{r} c_{2} h^{2}}{12 \alpha^{2}}$

\section{GRAPHICAL ILLUSTRATIONS}

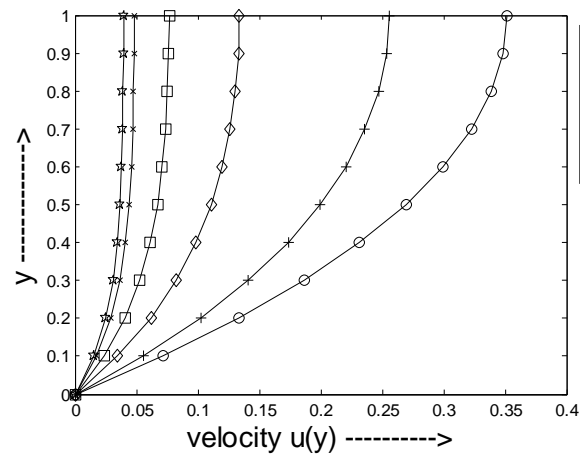

Fig. 1 velocity profile for $\mathrm{c} 1=1$ and $\mathrm{h}=1$

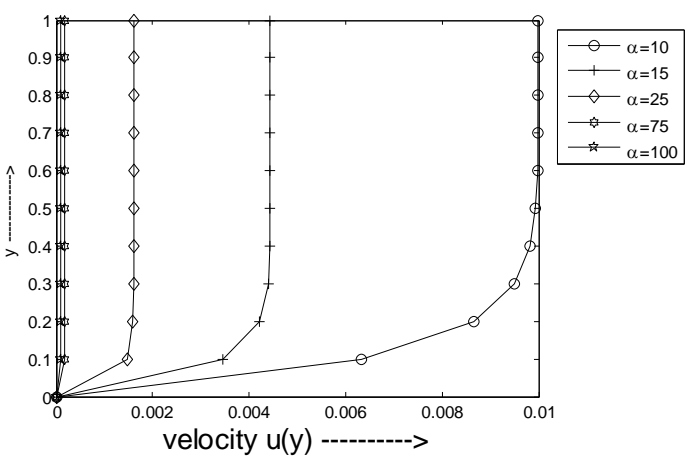

Fig.3. velocity profile for large $\alpha, c 1=1 \& h=1$

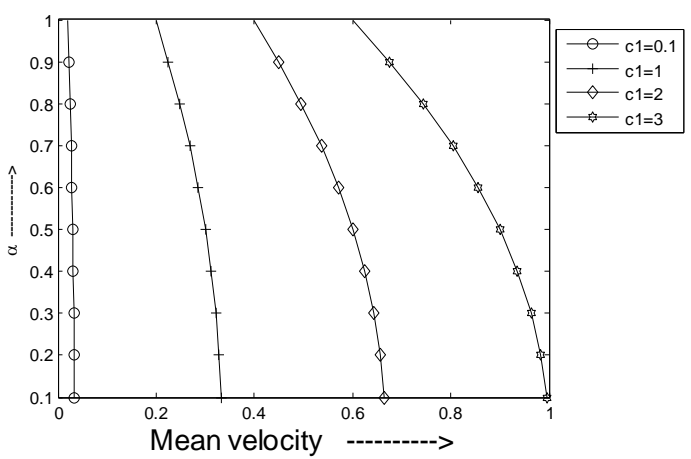

Fig.5. Mean velocity for small $\alpha \& h=1$

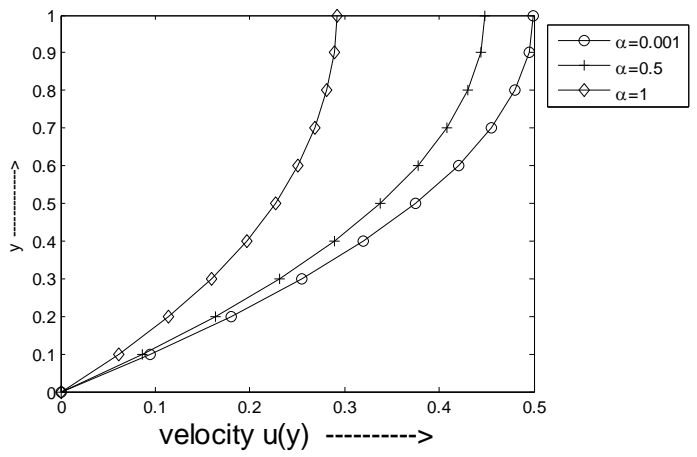

Fig.2.velocity profile for small $\alpha, c 1=1 \& h=1$

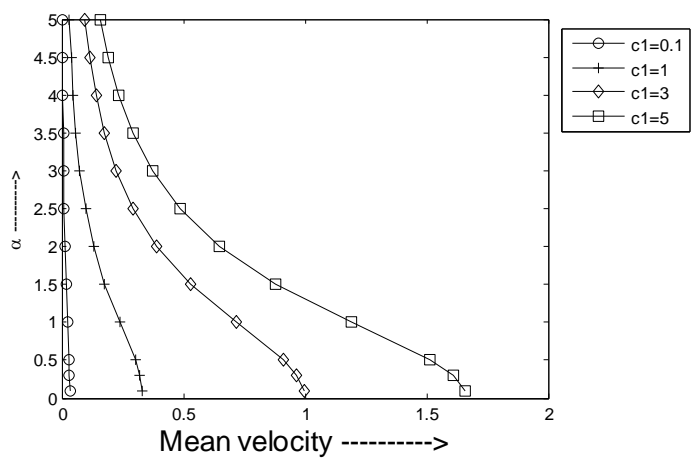

Fig. 4 mean velocity for $h=1$

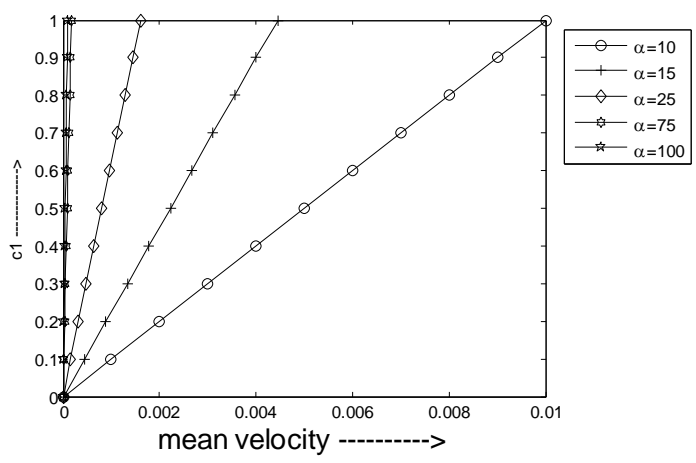

Fig.6. mean velocity for large $\alpha$ 


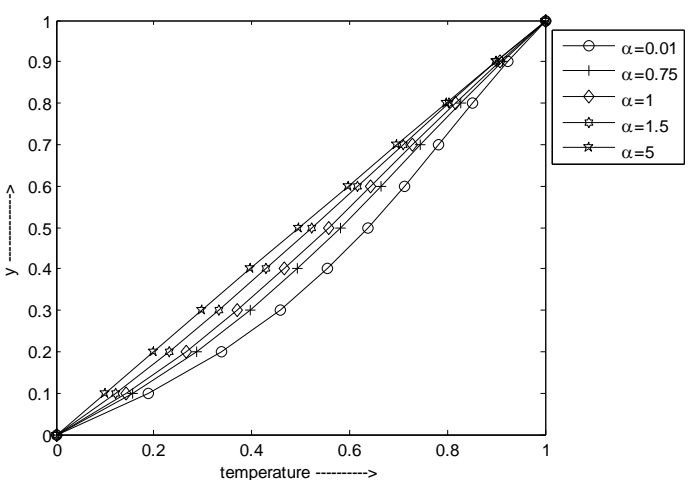

Fig.7. Temperature distribution for $\mathrm{h}=1, \mathrm{E}=5, \mathrm{c} 1=1, \mathrm{c} 2=1, \mathrm{p}=1$

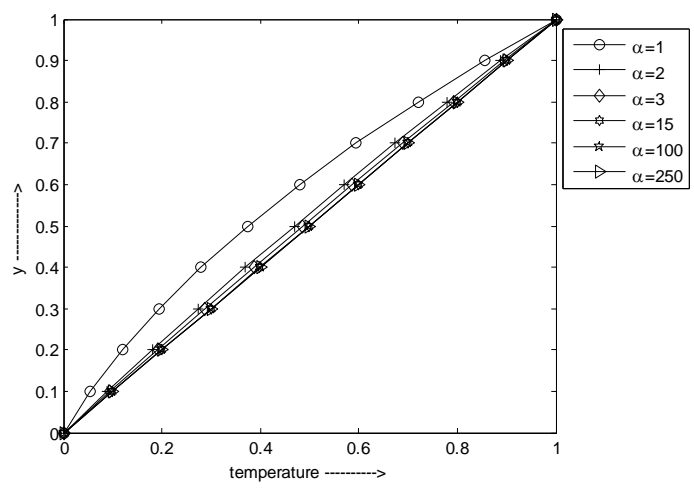

Fig.9. Temperature distribution for large $\alpha$ \& for $h=1, E=5, c 1=1, c 2=1, p=1$

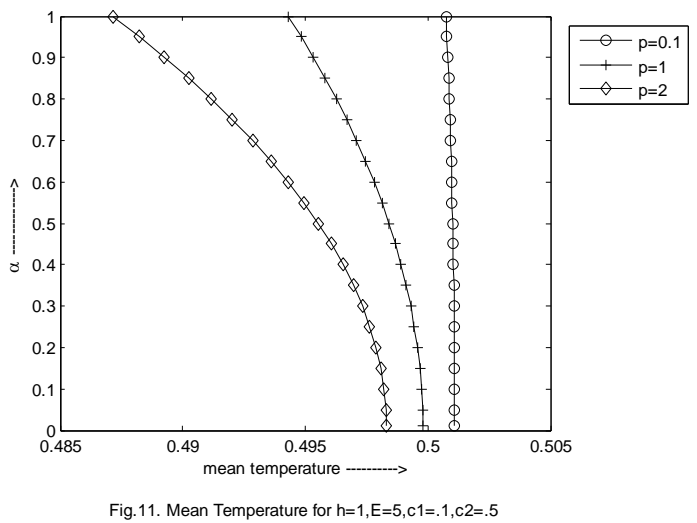

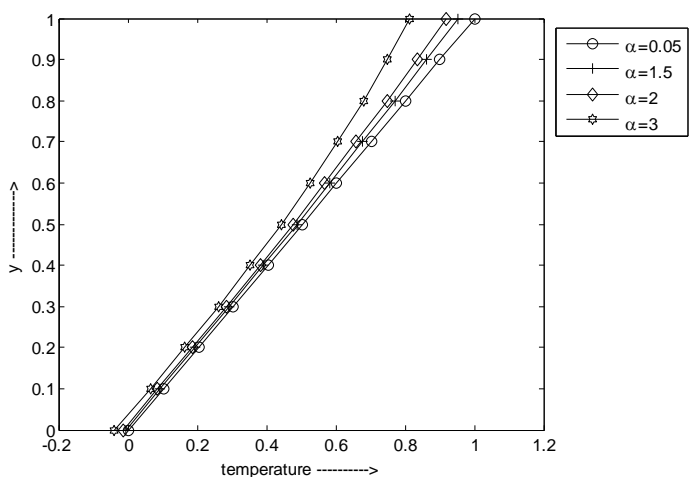

Fig.8. Temperature distribution for small $\alpha$ \& for $h=1, \mathrm{E}=5, \mathrm{c} 1=.1, \mathrm{c} 2=.5, \mathrm{p}=.1$
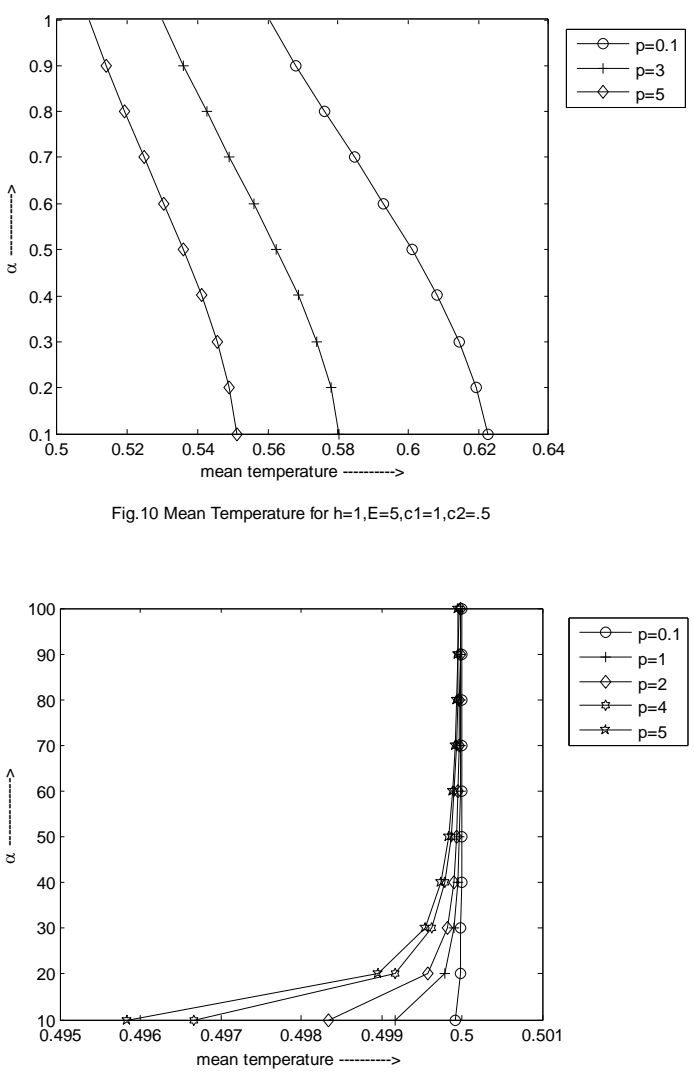

Fig. 12. Mean temperature distribution for large $\alpha$ \& for $h=1, \mathrm{E}=5, \mathrm{c} 1=1, \mathrm{c} 2=1$

\section{RESUlTS AND DISCUSSIONS}

It is noticed that the velocity profiles are more steep for large values of $\alpha$ that is the velocity of the fluid decreases with the increase in the value of $\alpha$ (Fig 1). From Fig.2, in the case of small $\alpha$, it is noticed that the velocity of the flow region decreases with the increase in the values of $\alpha$.For the case of large $\alpha$, Fig.3, illustrates that the velocity decreases with the increase in the values of $\alpha$ and tends to zero for the values of $\alpha$ bigger than 100 .

It is evident from the Fig.4, that for the increasing values of the pressure gradient $\mathrm{c} 1$ the mean velocity increases and appears to be decreasing with the increase in the values of $\alpha$. Fig.5(for the case of small $\alpha$ ), illustrates that the mean velocity is high for the higher values of the pressure gradient $\mathrm{c}_{1}$ at the bottom plate and decreases gradually with the increase in the values of $\alpha$. In the case of large $\alpha$, an increase in the values of $\alpha$ decreases the mean velocity of the flow region (Fig.6).

It is clearly illustrated in the Fig.7, that the temperature profiles gradually decreases with the increase in the values of $\alpha$. For the case of small $\alpha$, an increase in the value of $\alpha$ decreases the temperature in the flow region(Fig.8). From Fig.9, in the case of large $\alpha$, it is noticed that the temperature profile 
of the flow region increases with the increase in the values of $\alpha$,remains unaltered for large values of $\alpha$.

It is noticed that the mean temperature decreases as the prandtl number $\mathrm{P}$ increases along with the increase in the values of the porosity parameter $\alpha$. (Fig.10). In the case of small $\alpha$, Fig.11, illustrates that the mean temperature is high for smaller values of the prandtl numbers at the bottom plate and gradually decreases with the increase in the values of $\alpha$. Fig.12, in the case of large $\alpha$, illustrates that the mean temperature remains almost un altered for higher values of $\alpha$ and for lower values of $\alpha$ it decreases with the increasing prandtl number $\mathrm{p}$.

\section{REFERENCES}

[1] N.Ch .Raghava Charyulu, Study of fluid flows in porous and non-porous channel Ph.D Thesis, IIT Bombay (1984).

[2] G.Venkata Satya Narayana Raju, Some problems of fluid flows and heat transfer in porous and non-porous ducts Ph.D thesis (1989).

[3] Md.Ameenuddin and N.Ch.Pattabhi Ramacharyulu,Steady flow of a second order slightly viscous fluid through a porous medium between two horizontal permeable plates in relative motion, International Journal of Mathematical Sciences,Technology and Humanites,Vol 1,pp.1-18,(2011).

[4] Jaweed Ameen, Some investigations on fluid flows through generalized porous media, Ph.D Thesis, Banasthali University, (2009).

[5] A.A Raptis and C.P Perdikis, Oscillatory flow through a Porous medium by the presence of free convective flow,Int.J.Engg.Sci,Vol23,No1,pp 51-55( 1975).

[6] K.Yama Moto and N.Iwamura, Flow with convective acceleration through a porous medium, J. Physical society Japan,Vol. 37,No.3(1976), pp.41.

\section{AUTHOR's BIOGRAPHY}

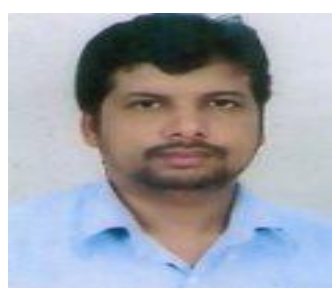

Dr. Khaja Moinuddin, is working as an Assistant Professor (Senior scale) in Mathematics at Maulana Azad National Urdu University, Gachibowli, Hyderabad. He has a total teaching experience of twenty six years at Under Graduate and Post Graduate levels. He has published 14 research articles in the Journals of National and International repute. His areas of interest are Porous Media, Algebra and Analysis

Citation: K. Moinuddin, " A Heat Transfer Problem of Viscous Liquid in Porous Media ", International Journal of Scientific and Innovative Mathematical Research, vol. 5, no. 8, p. 1-7, 2017., http://dx.doi.org/ 10.20431/2347-3142.0508001

Copyright: (C) 2017 Authors. This is an open-access article distributed under the terms of the Creative Commons Attribution License, which permits unrestricted use, distribution, and reproduction in any medium, provided the original author and source are credited. 